ration by Professor Matthews of Louisville-was done outside the sphinoter muscle. I have neglected to say that the sphincter was divulsed early in action, and it might be of interest to mention that a Matthews' speculum-that being the instrument usedwas broken in doing so, so strong was the sphincter muscle.

I shall not conjecture what would have been the outcome if I had left that second pint of pus in this patient a few hours longer.

\section{THE ARTHRITIC DIATHESIS.}

BY R. ALEXANDER BATE, A.B., M.D.

CHIFF OF THE MEDICAL CLINIC AND ASSISTANT TO THE CHAIR OF THE PRINCIPLES AND PRACTICE OF MEDICINE AND CLINICAL MEDICINE, HOSPITAL COLLEGE OF MEDICINE.
LOUISYILLE, KY.

The term "diathesis" is applied to an inherited predisposition to alterations of nutrition. Nutrition consists of the transformation of non-living molecules into living cells (assimilation) which undergo chemic changes with oxygen (reaction), producing waste material that in time is thrown off to make room for fresh cells (disassimilation). Nutrition is controlled by the nervous system, and is modified by age, sex, climate, food, geographic and geologic position, occupations, manners and customs, hygienic conditions, variations of light, heat, moisture and atmosphere (environment). Continuous subjection to any of these conditions modifying nutrition becomes apparent in the progeny of individuals; and this functional alteration of nutrition, causes a predisposition to pathologic conditions, or a pathologic condition itself, known as diathesis.

Bouchard defines "constitution" as including all that is concerned with the structure and framework of the body; while the word "temperament" includes everything that pertains to the individual characteristics and functional activity. Constitution and temperament are the gifts of heredity, and, either healthy or morbid, are transmitted from parent to progeny. Hence diathesis is morbid temperament.

Two diatheses are admitted: scrofula, or a predisposition to tuberculosis, and arthritism, or a predisposition to certain diseases characterized by retardation of the processes of nutrition. The latter is for our present consideration. The alterations in arthritism are purely functional, consequently there is no morbid anatomy. Since there are disturbances in which the retrograde changes produce a persistent increase in the products of disassimilation, such as uric and lactic acids; and since, in some instances, in spite of diet and exercise, these materials are formed, we must assume that in this diathesis there is an inability on the part of the cells to produce oxidation, even when the necessary articles are furnished.

The various manifestations of arthritism are those forms of arrested metamorphosis characterized by the presence or excess of organic acids. Some of the aoids are volatile, and are eliminated by the skin and lungs; some may remain in the blood or be stored in the tissues; some effect changes by increasing acidity, while others unite with bases and do not affect the relative reaction of the fluids. Consequently, we have the manifestations or symptoms of arthritism varying according to the stage at which metamorphosis is arrested and according to the character and consequent retention or channel of retention. Thus uric acid, the most prominent product of disassimilation, causes one class of diseases when it is in solution in the blood, and another totally different class when it is precipitated into the tissues.

The diseases generally conceded as dependent upon the arthritic diathesis are: acid dyscrasia, rickets, osteomalacia, obesity, lithiasis, embracing biliary, renal and pancreatic ; diabetes mellitus and insipidus; rheumatoid arthritis; articular and abarticular rheumatism; gout in all its phases; the constitutional insanities, anemia, eczema, neuralgia, migraine, arteriocapillary fibrosis; nasal, bronchial and cardiac asthma, hemorrhoids, biliousness, dyspepsia, paralysis, neurasthenia, hysteria, epilepsy, Reynaud's disease, albuminuria and Bright's disease.

In other words, as has been taught for some time, diseases may be divided into two classes, those having an external or foreign cause, as the microbic diseases, those having an internal or auto-cause, as those due to errors of metabolism. In the microbic type the oxygen is extracted from the blood by the foreign sources; in the metabolic type functional alteration or deficiency lessens oxygenation. Haig and Bouchard likewise strongly suggest that the action of drugs depends upon their power of increasing or diminish. ing this or that step of metabolism. Various forms of auto-intoxication are paralleled by the reaction from certain alkaloids.

In the first-mentioned manifestation of the arthritic diathesis-acid dyscrasia-there occurs during the process of oxidation a number of organic acids, such as uric, hippuric, oxaluric, oxalic, lactic and its derivatives, caprylic, caproic, valerianic, butyric, propionic, formic, acetic, stearic, oleic and palmitic, resulting from the incomplete oxidation of nitrogenous, saccharine, starchy and fatty substances, also from cholesterin, from the pancreatic juice and peptones and from glycocoll. Those formed in the alimentary canal, as lactic, its derivatives, etc., become absorbed and pass into the circulating liquids of the body and the tissues, where they undergo more or less trans. formation and elimination through the excretory organs. Carbonic and uric acids are usually combined with bases, and do not by their presence increase acidity in the tissues. Formic, acetic, butyric and several other volatile fatty acids are eliminated by the skin; butyric and cholalic also with the feces; renic, hippuric, oxaluric, carbonic, taurylic, damaluric, damalic, succinic and oxalic acids with the urine. Hydrochloric acid may exist in excess in the stomach, but more frequently the acidity is produced by the presence of some of the organic acids, as a consequence of fermentation induced by the presence of microphytic ferments.

In rachitis the primitive blood change is believed to be an excess of lactic acid, which holds the phosphate of calcium, etc., in solution, thus preventing the formation of infantile bone. Osteomalacia, on the other hand, occurs in the adult, and an excess of lactic acid dissolves the already formed bone. Obesity seems directly dependent upon oxidation. In addition to fat formed directly from food, there is a certain amount formed during retrograde metamorphosis, both of the nitrogenous and albuminoid constituents of the tissues. Obesity occurs in the anemic, on account of the diminished blood-current being insufficient to carry a normal amount of oxygen; likewise in the paralyzed, the nerve-cells being deficient. Oxidation of emulsified fat is more difficult than of the saponified, consequently arrest of the action of 
the pancreatic juice by the too acid contents of the stomach being passed into the intestine causes fat to be stored up. Diabetes mellitus we find alternating with obesity and following osteomalacia when the latter has received the benefit of improved hygiene or increased oxidation. In diabetes insipidus probably the nervous system is first affected by retention of uric acid, since we have the disease following lead and alcohol poisoning and sudden refrigeration of the body, which things Haig has shown favor the precipitation of uric acid by raising the acidity of the blood. Dilatation of the capillaries of the kidneys may be the immediate result of mechanic obstruction in the form of the precipitated uric acid in the arterioles.

In biliary lithiasis the cholesterin becomes precipitated from the bile, when the calcium salts unite with the organic acids to form insoluble salts. Renal calculi result directly from the acids, uric acid being the most frequent form. Pancreatic calculi are dependent upon the precipitation of the calcium salts from an acid medium.

Rheunatoid arthritis, according to Haig, is one stage of the same condition known as gout. He reported a postmortem at St. Bartholomew's Hospital in which Sir Dyce Duckworth observed rheumatoid changes, that is erosions without urates, and gouty changes or erosions with a deposit of urates in the joints, in the same cadaver. In the rheumatoid joint the urates had been dissolved out and eliminated in the urine prior to death. Rheumatism, gout and valvular disease of the heart are produced by the deposit of the urates in the tissues, which is caused by increased acidity of the blood. Asthma, cephalalgia, epilepsy, arterio-capillary fibrosis and all the other conditions are dependent upon uric-acidemia, which increases arterial tension and affects the in. terstitial circulation of the various organs and tissues.

In speaking of these diseases as dependent upon arthritism, it is not meant that no other causative factor exists, or that these diseases may not be acquired, but that the essential condition corresponds to the "arthritic diathesis." Bouchard says: "The reaction of a disturbed nervous system, by corrupting for the moment nutrition, can produce morbid opportunity, and may modify nutrition in a lasting manner and develop an acquired diathesis. The acquired diathesis, once established, may become transmissible." Horbaczewski has shown uric acid may be derived from nuclein. Haig believes a proportional amount of urea is also derived from nuclein, and the uric acid and urea are always formed in the system in the proportion of 1 to 35 or 40 . If a less proportion of uric acid is eliminated, it indicates that uric acid is being retained in the tissues. If a greater proportion is being eliminated, it indicates uric acid previously stored up is being dissolved out.

Since uric acid is so frequent a factor in disease, the treatment must embrace such measures as free uric acid from both the blood and the system. In general the treatment is dietetic, hygienic and medicinal. Haig says "the one thing needful is a proper diet," and restricts all red meats, coffee, tea and eggs. Breads, milk, vegetables, fruits and nuts are permitted.

In rickets and osteomalacia, however, that food richest in phosphates should be selected, that is eggs, fish, cracked wheat and oatmeal. Strawberries, tomatoes, bananas and excessive saccharine materials are generally harmful in the uric acid diseases.
The hygienic treatment consists of outdoor exercise, mountain.climbing, sea voyages, baths and massage, to increase oxidation. Flannels should be used to prevent sudden refrigeration of the surface. A dry, warm climate should be selected. The medicine should vary according to the individual type of the disorder. The salicyl group is the best to eliminate uric acjd from the system, the iodids to remove it from the blood and to lower arterial tension. Hence, the prognosis in such disorders as glycosuria, albuminuria, asthma, lithiasis and the arthropathies, when observed before morbid changes occur, is much better than the former uncertain results permitted. Cells once destroyed are not replaceable, but since, by exercise, diet and the uric acid solvents, we can prevent the further destruction of cells, the disease may in many instances be arrested.

Good results from antilithemic measures in the disorders of arthritism are reported by such observers as Lyman, Potter, Shoemaker, Wilcox, Oleve and Hunter, Bigelow, Satterthwaite and many others too numerous to mention. Personally, I have experienced favorable results from antilithemic remedies in glycosuria, nasal and bronchial asthma, lithiasis, albuminuria, obesity, eczema, paresis, rheumatism, angina pectoris, recurrent typhilitis, vertigo, biliousness, dyspepsia, neuralgia and migraine.

\section{THERAPEUTICS OF COAL-TAR PRODUCTS ${ }^{1}$.}

A CLINICAL REPORT.

Presented to the Section on Materia Medica, Pharmacy and Therapeutics, at the Forty-ninth Annual Meeting of the American Medical Association, held at Denver, Colo., June 7-10, 1898. BY W. R. INGE DALTON, M.D.

Member American Medical Association, New York County Association, Medical Society County of Kings, and Now York County Medical Society. NEW YORK CITY.

Agrescite medendo: "The medicine increases the disease," is an appropriate motto for the introduction of this paper, for by the unskillful exhibition of coaltar products in disease great harm has resulted. The immense and very extraordinary development of synthetic chemistry has exerted a profound influence upon the medical practice of recent times. The German school has inundated this side of the water with their synthetic compounds-legions of them have been introduced-we have been bombarded with dis. coveries of new organic compounds by the organic chemist, all purporting to possess marvelous and rare virtues, and foisted upon us with the brazen assurance that they open up vast possibilities of therapeutic application. Is it not time to call a halt upon the arrogant assumption of these foreign proprietors of synthetic drugs who dictate to us with such unblushing effrontery, and give the older medicine as chance? I lift my voice against the indiscriminate use of coal-tar products, and assert that more harm has been done by the use of antipyrin, phenacetin, laotophenin, acetanilid, etc., than any good derived by their exhibition. True, they reduce temperature, but at what expense to the economy! How do they exert such a reduction? Antipyretics do so, either by lessening the generation of heat, or by aiding the operation of its loss through radiation-the labor of evaporation of perspiration and conduction. Those remedies which lessen oxidation, which depress the 1 Original abstract of the paper read before the American Medical Association. 